\title{
Case Report: The Effects of Photobiomodulation (Modified Intravascular Laser Irradiation) on Quality Life, Clinical Symptoms, Reduction of Inflammatory Markers and Oxidative Stress in Schizophrenia
}

\author{
Viviane Batista Cristiano ${ }^{1,2 *}$, Michele Fonseca Szortyka ${ }^{1,2}$ and Paulo Belmonte-de-Abreu ${ }^{3}$ \\ ${ }^{1}$ Graduate Program in Psychiatry and Behavioral Sciences, Universidade Federal do Rio Grande do Sul, Brazil \\ ${ }^{2}$ Schizophrenia Program, Hospital de Clínicas de Porto Alegre, Brazil \\ ${ }^{3}$ Psychiatrist, Department of Psychiatry, Universidade Federal do Rio Grande do Sul Medical School Schizophrenia Program, Brazil
}

Submission: May 17, 2020; Published: July 09, 2020

*Corresponding author: Viviane Batista Cristiano, Graduate Program in Psychiatry and Behavioral Sciences, Universidade Federal do Rio Grande do Sul, Schizophrenia Program, Hospital de Clínicas de Porto Alegre, Porto Alegre, Rio Grande do Sul, Brazil

\begin{abstract}
Schizophrenia is a chronic mental disorder; these patients have life expectancy and comorbidities. They usually have different altered inflammatory and oxidative markers. Already Modified Intravascular Laser Irradiation of Blood (mILIB) is a Photobiomodulation technique with direct laser $(606 \mathrm{~nm})$ and continuously placed over the radial artery with a bracelet. We investigated the clinical effects of mILIB on inflammatory and oxidative markers and evaluated the therapeutic effect in patients with schizophrenia. Patient concerns: The 49-year-old male with DSM V diagnosis of Schizophrenia. He is a smoker, hypertensive and using medication (Risperidone). Intervention: Once the patient was in stable condition, 20 sessions of 30 minutes each, in the following order: 10 daily sessions of 30 minutes, followed by 20 days of interval, and 10 additional daily 30 minutes sessions. Instruments: quality of life using the SF-36 questionnaire, clinical symptoms using the BPRS questionnaire, Blood tests included high sensitivity C - reactive protein, high sensitivity troponin, lactate, and creatine kinase. After the mILIB protocol, the patient showed improvement in 6 domains in the assessment of quality of life, in the severity of the disease, the patient showed an improvement in the percentage of scale change, in addition to improvement in the inflammation marker and oxidative stress; only troponin presented the opposite result. It is an initial study from which we obtained favorable results for the patient with schizophrenia.
\end{abstract}

Keywords: Case report; Schizophrenia; Modified intravascular laser irradiation; Photobiomodulation; Inflammatory; Oxidative markers and quality of life

Abbreviations: SCZ: Schizophrenia; mILIB: Modified Intravascular Laser Irradiation of Blood; LLLT: low-level laser therapy; hs-CRP: High sensitivity C-reactive protein; hs-Troponin: high sensitivity troponin; LC: lactate; CK: Creatine Kinase; QLSF-36: Quality of life was assessed by SF-36; BPRS: Brief Psychiatric Rating Scale

\section{Introduction}

Schizophrenia (SCZ) is a mental disorder that affects around $1 \%$ of the world population and that generates progressive brain and body changes, which leads many authors to classify it as a systemic disease [1,2]. Patients with this diagnosis have short life expectancy, on average 20 years less than the general unaffected population [2]. A plausible explanation for this decrease in life expectancy is the deleterious effects of long-term drug treatment (including weight gain and metabolic alterations) and comorbidities such as diabetes mellitus and cardiovascular diseases that are more prevalent in this disease than in the general population [2]. They usually have different altered inflammatory and oxidative markers, suggesting a pro-inflammatory pattern of illness that may be directly associated both to disease on set, course and even symptoms [3]. Recently that have been several studies targeting inflammation, with drugs like minocycline, celecoxib, methotrexate, with variable results, but some with positive effects over brain atrophy and symptoms [4]. Additional 
anti-inflammatory therapeutics, photobiomodulation (PBM), acts at the cellular level by stimulating cell metabolism, mainly mitochondria, cytochrome $\mathrm{C}$ oxidase, increasing ATP synthesis, with clear anti-inflammatory actions [5]. Although evidencing effect in several inflammatory diseases (muscular and vascular), it was never used specifically in SCZ [5]. mILIB is a PBM technique with direct laser and continuously placed over the radial artery with a bracelet, with frequency and duration fitted according to specific pathology $(15,30$ and 60 minutes). Taking into account the pro-inflammatory and oxidative condition that SCZ generates in its patients, and in contrast the beneficial effects of PBM/ mILIB provides, we made an open-label study of add-on mILIB in a chronic patient with the diagnosis of schizophrenia that had indication of PBM due to its clinical and metabolic profile.

\section{Case report}

The subject was a male, 49 years old, caucasian; single, smoker, hypertensive with DSM V diagnosis of SCZ, followed at the SCZ Outpatient Clinic of a major University Hospital (Hospital de Clínicas de Porto Alegre) in Southern Brazil, under Public Health coverage. First episode was at the age of 18, after 3 years of cannabis use. The patient had 3 psychiatric hospitalizations and he was stabilized in the last 10 years. He uses medication (Risperidone). Patient and relatives signed a consent form, after receiving the explanation of the technique. mILIB consisted of 20 sessions of 30 minutes each, in the following order: 10 daily sessions of 30 minutes, followed by 20 days of interval, and 10 additional daily 30 minutes sessions. The patient received mILIB in the dorsal position, with a protective eyeglass, with light applied over radial artery (interspersed right/left) with a bracelet fitting the equipment Recover MM Optics $®$ (Red laser 660nm). The laser had a power of $100 \mathrm{~mW}$, providing $180 \mathrm{~J} /$ session. Quality of life was assessed by SF-36 (QLSF-36), and symptoms by Brief Psychiatric Rating Scale (BPRS). QLSF-36 has 8 domains (functional capacity, vitality, pain, mental health, limitations due to physical aspects, limitations due to emotional aspects, limitations due to social aspects and general health status), with a score from 0 to 100 (where 0 is the worst quality of life and 100 the best quality of life); BPRS was composed by 18 items, based on the patient's verbal information and on the observed behavior during the interview (where 0 is the best symptom and 6 the worst symptoms) and the blood samples were collected at the HCPA Clinical Pathology Service as per routine laboratory practice; hs-CRP, hs-Troponin, Lactate and Creatine Kinase.

\section{Results}

The patient followed all the protocol, without complications. Table 1 shows the results of the mILIB protocol in relation to quality of life using the QLSF-36. Of the 8 domains, 6 improved. Highlighting the emotional limitations domain that showed $100 \%$ improvement. Table 2 shows the results of the mILIB protocol in relation to clinic symptoms using the BPRS questionnaire. We can see that we got an improvement that ranged from 70 to $100 \%$ of the symptoms.

In Figure 1 we have the results of inflammatory markers and oxidative stress. hs-CRP presented a drop in 6,53\%, as well as the CK (drop of 51,20\%) and the LC (drop of 63,3\%). The hs-Troponin showed an increase of $14,76 \%$.
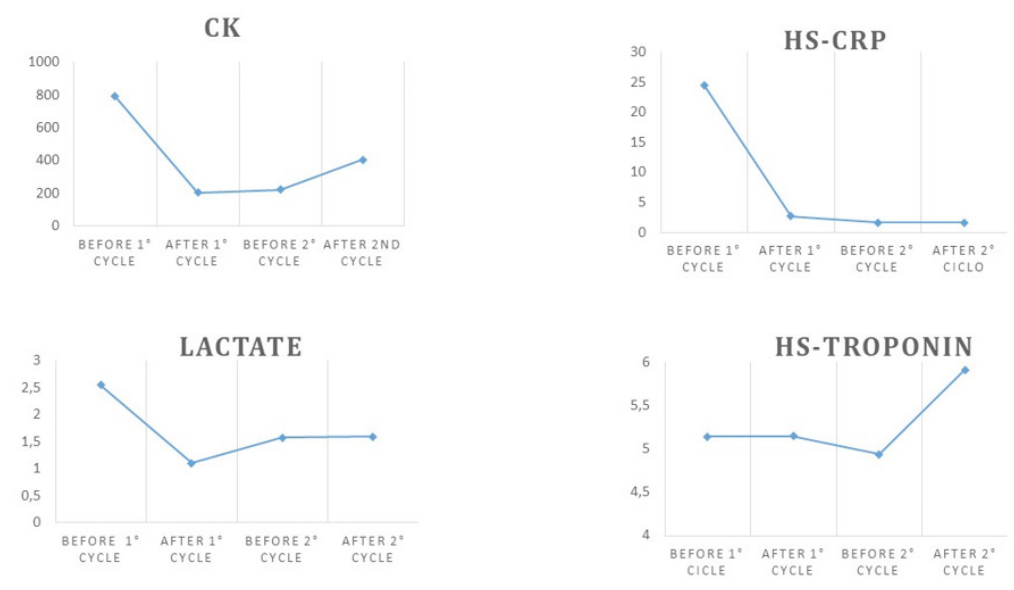

Figure 1: Display results of inflammatory and oxidative proteins in 4 moments of the patient with SCZ (day 1, before the 1st cycle, day 10 , after 1 st cycle, day 30 , before 2 nd cycle and day 40 , at the end of the 2 nd cycle).

$\mathrm{CK}=$ Creatine Kinase.

$\mathrm{Hs}-\mathrm{CRP}=$ high sensitivity $\mathrm{C}$-reactive protein.

Hs-Troponin= high sensitivity troponin. 


\section{Psychology and Behavioral Science International Journal}

Table 1: Changes over 2 cycles of PBM of a patient with SCZ.

\begin{tabular}{|c|c|c|c|c|}
\hline SF36 & Before & After & Difference & \%Difference \\
\hline Functional Capacity & 85 & 65 & -20 & 24 \\
\hline Physical Limitation & 100 & 75 & -25 & 27 \\
\hline Pain & 20 & 74 & 16 & 25 \\
\hline Mental Health & 64 & 80 & 25 & 48 \\
\hline General Health & 52 & 77 & 0 & 0 \\
\hline Vitality & 50 & 50 & 50 & 100 \\
\hline Social Life & 50 & 100 & & 27 \\
\hline Emotional Limitation & 0 & 100 & & 100 \\
\hline
\end{tabular}

Table 2: BPRS over 2 cycles of PBM of a patient with schizophrenia.

\begin{tabular}{|c|c|c|c|c|c|}
\hline BPRS Factors & BPRS Items & Before & After & Change & Change \\
\hline Anxiety/Depression & $5+2+9+1$ & 5 & 5 & 0 & 0.00 \\
\hline Withdraw/Isolation & $3+13+14+16+18$ & 6 & 0 & -6 & 100.00 \\
\hline Thought Disorder & $11+15+12+4+10$ & 5 & 0 & -5 & 100.00 \\
\hline Activation & $7+6+17+8$ & 5 & 1 & -4 & 80.00 \\
\hline Total & $1-18$ & 20 & 6 & -14 & 70.00 \\
\hline
\end{tabular}

\section{Discussion}

The clinical and biochemical changes observed in this case of a man with SCZ maintained in stable medication that is in line with recent PBM studies in patients with depression, Alzheimer and other types of dementia [5]. Two cycles of PBM evidenced significant changes not only in inflammatory markers, but also in psychotic symptoms. This observed effect if repeated in large series of patients and further in randomized controlled studies may put PBM as add-on treatment for patients with mental disorders such as SCZ early. These are promising findings, with improvement not only in metabolic parameters, but also in psychopathology and quality of life. Confirming a very broad effect of systemic PBM (mILIB). Psychopathology improvement was on three dimensions: Delay-isolation, Thought Disorder and Activation. Clinically, this would mean reduced isolation from others, more organized thinking, and increased energy. Additionally, beneficial effect in six of the eight different dimensions of quality of life, social, family and health functioning improved with PBM. Hs-CRP was the most significant metabolic change. The drop was constant in both cycles, even after maintained in the interval period. LC, on the other hand, showed a considerable drop at the end of the first cycle, reaching a plateau of normality after the second application cycle. CK was extremely high at the beginning of the study, with exponential drop at the end of the first cycle, stability along the interval and further increase at the end of the second cycle. Since CK increases with physical activity, and the patient reported increased physical activity after the first part of treatment, this could explain the effect in CK $[6,7]$. Hs-Troponin dropped at the end of the first cycle, reached a plateau during pause and increased at the end of the second cycle probably accompanied the increase in CK, since hs-troponin is also a marker of muscle damage $[7,8]$.

Recent studies with ILIB used different protocols in different pathologies $[9,10]$. Although the protocols vary from 10 to 30 sessions, with 30 to 60 minutes of application. The wavelength used was the same as our study $(660 \mathrm{~nm})$, as well as the continuous mode. The results of these studies are promising because, through ILIB therapy, they were able to reduce systolic and diastolic pressure in patients with systemic arterial hypertension [10], and in cerebellar injury after stroke, they managed to accelerate functional recovery [9]. Associating these results with our findings, we can verify the potential of the technique in different pathologies. This is the first case report using photobiomodulation in this pathology in which they showed an expressive and important clinical improvement for this population that have limited access to general health care and fewer opportunities for prevention and treatment that would be expected in a non-psychiatric population. But we have limitations such as being just one case, possible transition effect, and for the success of this treatment we need more studies on MILIB in SCZ and other diagnoses.

\section{Acknowledgement}

This study was financed in part by the "Coordenação de Aperfeiçoamento de Pessoal de Nível Superior - Brasil (CAPES)" Finance Code 001 and data collection and processing from FIPE/ HCPA. 


\section{Conflict of Interest}

The authors declare that the research was conducted in the absence of any commercial or financial relationships that could be construed as a potential conflict of interest.

\section{References}

1. Girdler SJ, Confino JE, Woesner ME (2019) Exercise as a Treatment for Schizophrenia: A Review. Psychopharmacol Bull 49(1): 56-69.

2. McGrath J, Saha S, Chant D, Welham J (2008) Schizophrenia: a concise overview of incidence, prevalence, and mortality. Epidemiol Rev 30: 67-76.

3. Müller N (2018) Inflammation in Schizophrenia: Pathogenetic Aspects and Therapeutic Considerations. Schizophr Bull 44(5): 973-982.

4. Chaves C, Marque CR, Maia-de-Oliveira JP, Wichert-Ana L, Ferrari TB, et.al. (2015) Effects of minocycline add-on treatment on brain morphometry and cerebral perfusion in recent-onset schizophrenia. Schizophr Res 161(2-3): 439-445.
5. Salehpour F, Mahmoudi J, Kamari F, Sadigh-Eteghad S, Rasta SH, et al. (2018) Brain Photobiomodulation Therapy: a Narrative Review. Mol Neurobiol 55(8): 6601-6636.

6. Ferraresi C, Huang YY, Hamblin MR (2016) Photobiomodulation in human muscle tissue: an advantage in sports performance? J Biophotonics 9(11-12): 1273-1299.

7. Schwartz JG, Prihoda TJ, Stuckey JH, Gage CL, Darnell ML (1988) Creatine kinase MB in cases of skeletal muscle trauma. Clin Chem 34(5): 898-901.

8. Shave R, Baggish A, George K, Wood M, Scharhag J, et al. (2010) Exercise-induced cardiac troponin elevation: evidence, mechanisms, and implications. J Am Coll Cardiol 56(3): 169-176.

9. Wan-Hua Y, Shiou-Ping L, Shin-Tsu C (2017) Case report: Rapid improvement of crossed cerebellar diaschisis after intravascular laser irradiation of blood in a case of stroke. Medicine 96: 2(e5646).

10. Jordão I, Silva J, Silva T, Rodrigues M, Horliana A, et al. (2019) Effect of irradiation with intravascular laser on the hemodynamic variables of hypertensive patients Study protocol for prospective blinded randomized clinical trial. Medicine 98: 14(e15111).

\section{Your next submission with Juniper Publishers will reach you the below assets}

- Quality Editorial service

- Swift Peer Review

- Reprints availability

- E-prints Service

- Manuscript Podcast for convenient understanding

- Global attainment for your research

- Manuscript accessibility in different formats ( Pdf, E-pub, Full Text, Audio)

- Unceasing customer service

Track the below URL for one-step submission https://juniperpublishers.com/online-submission.php 